\title{
Associação entre a técnica de análise de conteúdo e os ciclos de codificação: possibilidades a partir do softwareATLAS.ti
}

\author{
Dilmeire Sant’Anna Ramos Vosgerau', Dagmar Heil Pocrifka² ${ }^{2}$ Michele Simonian ${ }^{3}$ \\ dilmeire.vosgerau@pucpr.br, dagheil@gmail.com, michelesimonian@gmail.com \\ ${ }^{1}$ Pontifícia Universidade Católica do Paraná, Rua Imaculada Conceição 1155, 80215-901, Curitiba, Brasil \\ ${ }^{2}$ Universidade Federal de Pernambuco, Avenida Acadêmico Hélio Ramos s/n, 50670-901, Recife, Brasil \\ ${ }^{3}$ Universidade Federal do Paraná, Rua General Carneiro 460, 80.060-150, Curitiba, Brasil
}

DOI: 10.17013/risti.19.93-106

\begin{abstract}
Resumo: Este artigo analisa a possibilidade de associação de diferentes técnicas de análise quando, em um mesmo projeto,há fontes diversas de dados, como entrevistas, produções escritas e questionários, que produzem informação qualitativa. Associamos a técnica de análise de conteúdo (Bardin, 2010) aos ciclos de codificação (Saldaña, 2013), aplicando o software ATLAS.ti. O estudo revela que essa associação potencializa a criatividade metodológica dos pesquisadores e permite novas descobertas por meio da articulação das diferentes possibilidades de codificação, respondendo, com mais detalhes qualitativos, às questões de pesquisa. Além disso, destacamos que a aplicação dos indicadores sugeridos por Bardin (2010) nos dois ciclos de codificação propostos por Saldaña (2013) permite melhor sistematização do processo de codificação, diminuindo a subjetividade, devido às etapas e aos critérios claramente enunciados.
\end{abstract}

Palavras-chave: pesquisa qualitativa; análise de dados qualitativos; análise de conteúdo; ATLAS.ti.

\section{Association between the technical analysis of the content and the cycles of codification: possibilities from Atlas.ti software}

\begin{abstract}
This article analyses the possible association of different techniques of analysis, when there are several sources of data in the same project, such as interviews, written productions and quizzes, which produce qualitative information. It is done by the association of the technique of content analysis (Bardin, 2010) alongside with the cycles of codification (Saldaña, 2013) applied in the ATLAS.Ti. software. The study revealed that this association potentiates the methodological creativity of the researchers and allows new discoveries through articulation of different possibilities of codification, answering with greater qualitiative details the matters of the research. Besides, we highlight that, the application of these indexes suggested by Bardin (2010) in both cycles of codification proposed by Saldaña (2013) allowed a better systematization of the process of codification, lessening the subjectivity, due to the steps and criteria clearly enunciated.
\end{abstract}

Keywords: qualitative research; qualitative data analysis; content analysis; Atlas.TI. 


\section{Introdução}

Ao longo de seu processo de formação, os investigadores qualitativos passam por diversos dilemas e desafios (Alarcão, 2014; Leite et al., 2014), muitos dos quais podem ser solucionados na relação estabelecida com o orientador, que, tradicionalmente, faz a mediação presencial. Atualmente, no entanto, dispomos de recursos tecnológicos online que podem ampliar o apoio dado ao futuro pesquisador, caso do software on-line de supervisão da investigação (IARS) ${ }^{1}$ (Souza, Souza, Alarcão \& Moreira, 2015).

Mesmo com recursos de acompanhamento da pesquisa sofisticados, persistem aprendizagens essenciais que o investigador precisa construir, por exemplo, o aprofundamento metodológico necessário para garantir a qualidade e o rigor da pesquisa em andamento, de forma que os conhecimentos metodológicos possam ser a base para uma longa carreira de pesquisador e não para o desenvolvimento uma só pesquisa.

Dentre esses conhecimentos, estão aqueles que se referem aos métodos e às técnicas para a análise de dados qualitativos, que muitas vezes são apresentados aos estudantes como um labirinto de possibilidades e paradigmas; por exemplo: como codificar e categorizar os dados coletados para realizar descobertas, indo além das teorias que fundamentam a pesquisa? Como ser criativo e, ao mesmo tempo, preciso, para que a codificação e a categorização não estejam incongruentes com o que de fato os dados revelam? Quantas formas possíveis de codificar existem para além da decisão de ser um processo a priori, com códigos já estabelecidos, numa perspectiva dedutiva? Ou a posteriori, com os códigos sendo elaborados a partir do que os dados revelam, numa perspectiva indutiva (Saldaña, 2013)? Se o último paradigma for a escolha, como se pode codificar respeitando a subjetividade do pesquisador qualitativo, sem perder a fidedignidade e a ética ao tratar os dados produzidos em campo? A saída desse labirinto torna-se mais difícil ainda quando se questiona: quais caminhos são mais adequados a seguir nas análises de dados? E ainda: por que, em uma sociedade cibercultural, se é "malvisto" academicamente quando se defende a complementação e/ou substituição do tratamento de dados qualitativos pelas tradicionais técnicas de bricolagem? E, algumas vezes, por planilhas eletrônicas de softwares específicos para esse tipo de análise (Lage \& Godoy, 2008)? Nesse caso, considera-se que esses softwares podem auxiliar na sistematização das decisões assumidas em uma pesquisa qualitativa e, com isso, "reforçar a validade científica de tal escolha" (Teixeira, Mayr, Paisana \& Vieira, 2014, p. 97).

Diante desses questionamentos, com o intuito de aprofundar os conhecimentos sobre o tratamento e análise de dados qualitativos, este artigo amplia outro apresentado no $5^{\circ}$ Congresso Ibero-Americano em Investigação Qualitativa (CIAIQ2016) (Vosgerau, Pocrifka \& Simonian, 2016), que aborda alternativas oferecidas para a análise de dados qualitativos por meio da análise de conteúdo complementada por ciclos de codificação, aplicadas ao software ATLAS.ti.

A partir dessas reflexões, surge a seguinte indagação: de que forma as etapas da análise de conteúdo de Bardin (2010) podem ser complementadas pelos ciclos de codificação de Saldaña (2013) e implementadas com o auxílio do software de análise

${ }^{1}$ O IARS, desenvolvido a partir do guia de orientação de construção de plano de investigação elaborado por Isabel Alarcão, orienta o processo de desenvolvimento de pesquisa acadêmica. 
de dados qualitativos ATLAS.ti? Com essa questão, pretendemos contribuir com outros pesquisadores, apresentando alternativas para estabelecer critérios de escolha de estratégias de tratamento e análise de dados qualitativos, evitando assim, o desgaste e a perda de tempo no "labirinto sem saída" que pode se tornar esse processo.

Para tanto, este artigo é composto por quatro tópicos, contemplando este primeiro (Introdução) o contexto e a finalidade da pesquisa empreendida. Em Pressupostos teóricos, apresentamos as opções metodológicas de análise de dados qualitativos discutidas neste trabalho. Em Encaminhamento metodológico, descrevemos o cada etapa do processo de análise de dados, associando as duas técnicas apresentadas. Por fim, em Conclusões, respondemos à questão de pesquisa elaborada e apresentamos as conclusões feitas a partir da experiência sobre a análise de dados qualitativos.

\section{Pressupostos teóricos}

As possibilidades de uso do software ATLAS.ti têm sido amplamente discutidas em diferentes artigos acadêmicos (Varguillas, 2006; Vosgerau, Meyer \& Contreras, 2016), sob perspectivas diversas. Neste artigo, complementamos a proposta de uso dos recursos disponíveis no software descrita por Varguillas (2006), associando aos recursos duas proposições metodológicas de codificação de dados desenvolvidas por pesquisadores de repercussão internacional: a técnica de análise de conteúdo de Bardin (2010), publicada em 1970 e amplamente utilizada no contexto brasileiro, e os ciclos de codificação de Saldaña (2013), cuja obra, editada pela primeira vez em 2009, já está em sua terceira edição, totalizando oito reimpressões ao longo dos anos. Enquanto a proposta de análise de conteúdo (Bardin, 2010) tem sido associada a questões de pesquisa de diferentes naturezas, a escolha das técnicas descritas por Saldaña (2013) tem sempre como premissas a natureza e a questão de pesquisa propostas pelo pesquisador.

Assim comoVarguillas (2006), que, em seu artigo, buscou propor algumas recomendações pessoais à estratégia de análise de conteúdo, avançamos nessas recomendações ao estabelecer melhor detalhamento das possibilidades existentes para a codificação em dois ciclos, ou seja, a síntese entre análise de conteúdo e codificação em ciclos.

\subsection{O processo de análise proposto por Bardin (2010)}

Bardin (2010) apresenta a análise de conteúdo como técnica da análise qualitativa, proposta elaborada por volta da década de 1970, partindo de três processos, ou fases, entendidos como necessários para realizar uma análise de conteúdo: 1) pré-análise, 2) exploração do material e 3) tratamento dos resultados, inferência e interpretação (Figura 1).

A pré-análise dá origem à constituição do corpus da pesquisa, por meio da organização do material a ser analisado, tornando-o operacional. Ao mesmo tempo, ocorre a sistematização de ideias preliminares. Essa organização também possui um protocolo de quatro etapas: a leitura flutuante (etapa a), na qual se estabelece o contato com os documentos coletados e se busca um entendimento do material que o pesquisador tem em seu poder, para então realizar a escolha dos documentos (etapa b), que consiste na delimitação do que será analisado; por meio dessa leitura, ocorre a formulação das 


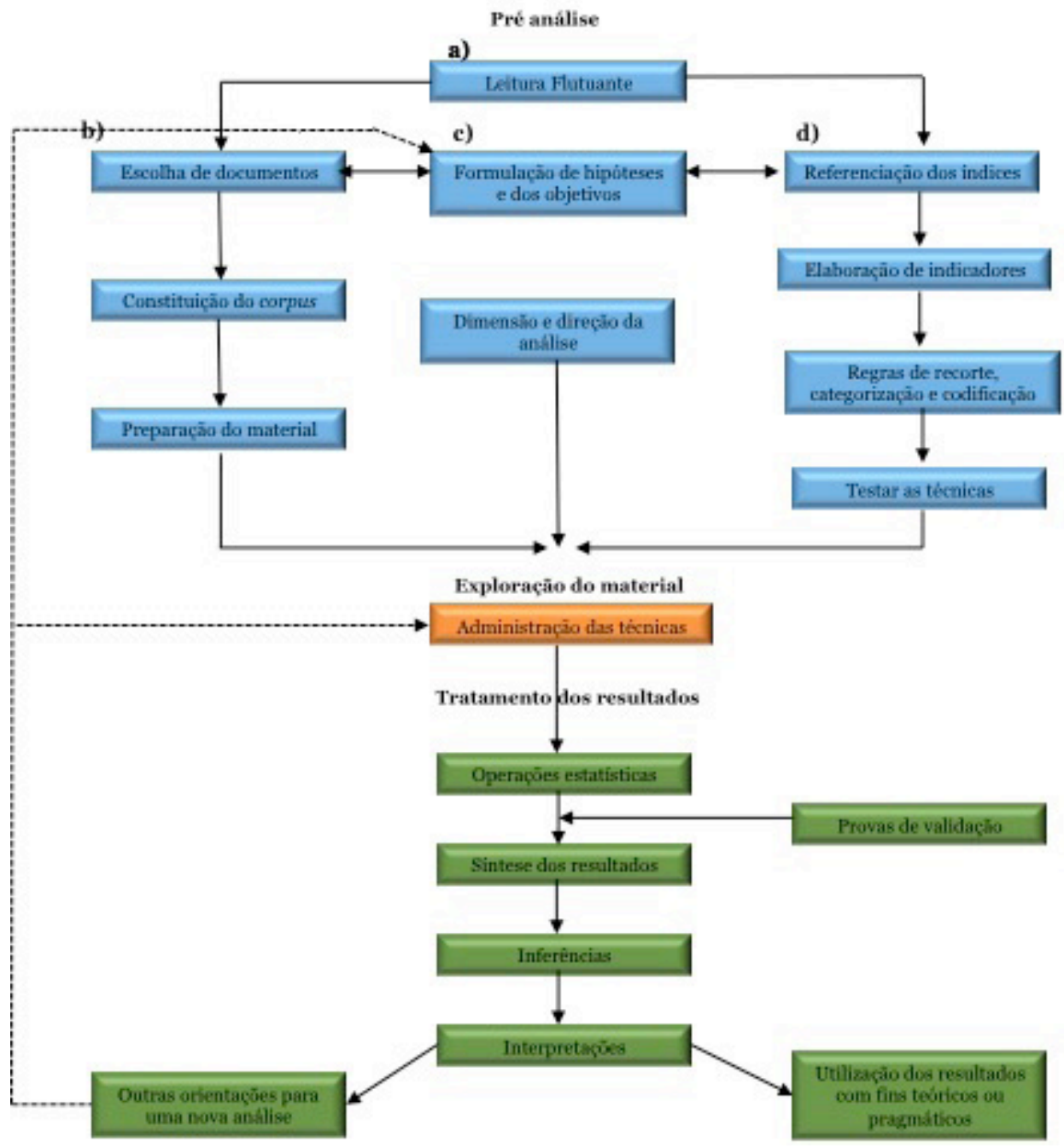

Figura 1 - Etapas da análise de dados qualitativos de Bardin (2010)

hipóteses e dos objetivos (etapa c), e também a referenciação dos índices e elaboração de indicadores (etapa d), envolvendo a determinação de indicadores por meio de recortes de texto nos documentos de análise (Bardin, 2010).

$\mathrm{Na}$ exploração do material, que representa a segunda fase, são administradas as técnicas de codificação do corpus, compreendendo o exame minucioso do material para a definição de categorias (sistemas de codificação) e a identificação das unidades de registro (unidade de significação a codificar, corresponde ao segmento de conteúdo a considerar unidade-base, visando à categorização e à frequência) e de contexto (unidade de compreensão para codificar a unidade de registro que corresponde ao segmento 
da mensagem, a fim de compreender a significação exata dela) nos documentos. Essa exploração do material é fundamental para o sucesso da pesquisa, pois pode viabilizar ou não a riqueza das interpretações e inferências. É considerada a fase da descrição analítica, a qual diz respeito ao corpus (todo e qualquer material textual coletado) submetido a um estudo detalhado, orientado pelas hipóteses e referenciais teóricos. Dessa forma, a codificação, a classificação e a categorização são elementos necessários nessa fase (Bardin, 2010).

A terceira fase diz respeito ao tratamento dos resultados, à inferência e à interpretação. Nela, os resultados são tratados e ocorre a condensação dos dados codificados, buscando as informações para análise, o que resultará nas interpretações inferenciais. É o momento de intuição, de análise reflexiva e crítica (Bardin, 2010).

\subsection{O processo de análise proposto por Saldaña (2013)}

Saldaña (2013) enfatiza que o processo de codificação é um dos caminhos da análise qualitativa de dados, mas não o único caminho. Isso porque, para o autor, todo o processo está relacionado ao campo da pesquisa, às opções ontológico-epistemológicas, às opções teóricas e aos recortes conceituais.

A importância de sua contribuição consiste na proposição de dois ciclos de codificação, com 31 possibilidades diferenciadas de elaboração de códigos. Para o autor, a codificação

\begin{tabular}{|c|c|c|}
\hline \multicolumn{3}{|c|}{ Primeiro ciclo de codificação } \\
\hline Método gramatical & Método elementar & Método afetivo \\
\hline $\begin{array}{l}\text { Codificação por atributo } \\
\text { Codificação por magnitude } \\
\text { Subcodificação } \\
\text { Codificação simultânea }\end{array}$ & $\begin{array}{l}\text { Codificação estrutural } \\
\text { Codificação descritiva } \\
\text { Codificação in vivo } \\
\text { Codificação de processo } \\
\text { Codificação inicial }\end{array}$ & $\begin{array}{l}\text { Codificação de emoções } \\
\text { Codificação de valores } \\
\text { Codificação de versos } \\
\text { Codificação de avaliação }\end{array}$ \\
\hline Método literário e de linguagem & Método exploratório & Método procedimental \\
\hline $\begin{array}{l}\text { Codificação dramatúrgica } \\
\text { Codificação de motivo } \\
\text { Codificação de narrativa } \\
\text { Codificação de diálogos }\end{array}$ & $\begin{array}{l}\text { Codificação holística } \\
\text { Codificação provisória } \\
\text { Codificação de hipóteses }\end{array}$ & $\begin{array}{l}\text { Codificação de protocolos } \\
\text { Esboço de materiais culturais } \\
\text { Codificação de domínios e } \\
\text { taxionomias } \\
\text { Codificação de causalidade }\end{array}$ \\
\hline \multicolumn{3}{|c|}{ Ciclo de transição entre o primeiro e o segundo } \\
\hline \multicolumn{3}{|c|}{ Codificação eclética } \\
\hline \multicolumn{3}{|c|}{ Segundo ciclo de codificação } \\
\hline & $\begin{array}{l}\text { Codificação de padrões } \\
\text { Codificação focada } \\
\text { Codificação axial } \\
\text { Codificação teórica } \\
\text { Codificação elaborativa } \\
\text { Codificação longitudinal }\end{array}$ & \\
\hline
\end{tabular}

Tabela 1 - Ciclos de codificação de Saldaña (2013) 
é um processo transitório entre o de produção dos dados e a análise extensiva destes, devendo, portanto, ocorrer de forma cíclica, e buscando o refinamento cada vez maior dos resultados encontrados (Saldaña, 2013). Na Tabela 1, apresentamos os ciclos descritos por Saldaña (2013), de forma a ampliar as possibilidades e favorecer a criatividade da codificação pelos pesquisadores, visando a obter respostas mais apropriadas para a questão de pesquisa proposta.

Antecedendo o primeiro ciclo de codificação, Saldaña (2013) propõe uma etapa de précodificação, na qual o pesquisador faz uma leitura inicial dos dados e elabora reflexões iniciais sobre o material coletado, denominadas memos ou memórias. Nessa etapa, as informações relevantes são sublinhadas, negritadas ou circuladas, não sendo necessária uma padronização no tamanho do fragmento, podendo ser assinaladas palavras e/ou frases que merecem atenção como chaves de evidência que embasam as suposições e se relacionam a teoria. Portanto, a elaboração de memórias (memos) analíticas contribui para a elaboração de questionamentos e o levantamento de causas e efeitos, análises pessoais e teóricas acerca do que foi destacado durante a leitura inicial.

A partir do tipo de pesquisa conduzido (etnográfico, estudo de caso, fenomenológico ou outro), associado à questão de pesquisa, o pesquisador escolhe um método ou uma combinação entre os 24 métodos indicados para o primeiro ciclo que mais favoreçam a resposta à questão de pesquisa.

Os métodos gramaticais favorecem a organização e categorização de informações demográficas quando utilizada a codificação por atributos. Ressaltamos que são aqueles que poderão auxiliar os cruzamentos dos dados codificados, de modo que Saldaña (2013) sugere sua utilização associada aos demais métodos escolhidos a partir da questão de pesquisa proposta. Já a codificação por magnitude permite classificar os participantes da pesquisa; por exemplo, em uma situação de experiência profissional, os participantes mais experientes e os menos experientes. Na técnica denominada subcodificação, o pesquisador pode definir que alguns códigos serão criados de modo categorizado; por exemplo, a cor dos olhos dos participantes poderia ser codificada como cor-dosolhos-azuis, cor-dos-olhos-negros, cor-dos-olhos-castanhos, cor-dos-olhos-verdes, cordos-olhos-não-definida. Por sua vez, a codificação simultânea permite ao pesquisador descobrir correlação entre as codificações realizadas, sendo utilizada quando, ao mesmo fragmento de texto, podem ser atribuídos dois códigos diferentes, pois em um mesmo parágrafo o participante apresenta informações que se relacionam.

Entre o primeiro e o segundo ciclos, situa-se o ciclo intermediário, no qual se busca compreender os resultados que emergem das codificações realizadas. As memos criadas na etapa de pré-codificação são fontes importantes para as análises realizadas nesta etapa. Saldaña (2013) recomenda que, a partir da codificação elaborada, os dados sejam reanalisados, gerando representações gráficas, por meio de tabelas de correlação entre códigos, mapas conceituais, mapas mentais, gráficos e esquemas, que auxiliem o pesquisador a chegar a possíveis respostas para a questão proposta. Uma vez que essa representação gráfica pode levar o pesquisador a refinar ainda mais o processo de codificação, o autor sugere, no segundo ciclo de codificação, mais seis métodos, que também devem ser escolhidos a partir da questão de pesquisa. 
A partir da análise das propostas de Bardin (2010) e Saldaña (2013), elaboramos o quadro comparativo entre as duas opções de codificação (Tabela 2).

\begin{tabular}{|c|c|c|}
\hline Autores & Bardin & Saldaña \\
\hline Ano (1 ${ }^{a}$ edição) & 1970 & 2009 \\
\hline Local & França & Estados Unidos \\
\hline Etapas & $\begin{array}{l}\text { 1. Pré-análise } \\
\text { 2. Exploração do } \\
\text { material } \\
3 . \text { Tratamento dos } \\
\text { resultados, inferência } \\
\text { e interpretação }\end{array}$ & $\begin{array}{l}\text { Pré-codificação } \\
\text { Anotações preliminares } \\
\text { Memos analíticas } \\
\text { Início do primeiro ciclo e escolha do estilo de codificação } \\
\text { Pode ou não ocorrer o segundo ciclo }\end{array}$ \\
\hline $\begin{array}{l}\text { Ordem das } \\
\text { etapas }\end{array}$ & $\begin{array}{l}\text { Sim, é necessária a } \\
\text { sequência ordenada } \\
\text { das etapas. }\end{array}$ & $\begin{array}{l}\text { Não necessariamente. Depende do objetivo a ser alcançado } \\
\text { na análise, bem como do recorte teórico, ontológico, } \\
\text { epistemológico e conceitual da pesquisa. }\end{array}$ \\
\hline Ciclos & $\begin{array}{l}\text { Textual } \\
\text { Conceitual }\end{array}$ & $\begin{array}{l}\text { Primeiro ciclo, com } 24 \text { possibilidades de codificação } \\
\text { Ciclo de transição } \\
\text { Segundo ciclo, com seis possibilidades de codificação }\end{array}$ \\
\hline $\begin{array}{l}\text { Recomenda } \\
\text { uso de } \\
\text { softwares }\end{array}$ & $\begin{array}{l}\text { Sim, mas os exemplos } \\
\text { apresentados associam } \\
\text { apenas a tratamento } \\
\text { estatístico. }\end{array}$ & $\begin{array}{l}\text { Sim. Os exemplos apresentados auxiliam a organização dos } \\
\text { códigos no software. No entanto, recomenda, anteriormente, } \\
\text { a aprendizagem do processo de forma manual, além de não } \\
\text { arriscar o uso sem treinamento prévio. }\end{array}$ \\
\hline
\end{tabular}

Tabela 2 - Comparativo entre Bardin (2010) e Saldaña (2013)

A elaboração do quadro comparativo auxiliou na tomada de decisões para a análise de dados, utilizando o software ATLAS.ti no estudo exploratório realizado.

\section{Encaminhamento metodológico}

Trata-se de um estudo exploratório, com o objetivo de conhecer o fenômeno, e de caráter descritivo (Richardson, 1999), pois busca a relação e a conformidade da análise qualitativa nas percepções de Bardin (2010) e Saldaña (2013).

O campo empírico consistiu em um seminário semestral, ministrado no Programa de Pós-Graduação em Educação da Pontifícia Universidade Católica do Paraná, visando a apresentar, deforma conceitual eprática, a análise dedados qualitativosinstrumentalizada pelo uso do software ATLAS.ti. Como atuantes nesse campo, participaram da pesquisa 25 estudantes de diferentes programas de pós-graduação da instituição e de outras universidades, que, estando inscritos em programas de pós-graduação stricto sensu, por sugestão da Comissão de Aperfeiçoamento de Pessoal do Nível Superior (Capes), podem cursar disciplinas em qualquer programa de pós-graduação do país. Do total de 25 participantes, 21 eram mulheres e quatro, homens, com variação de idade entre 21 e 57 anos. Ainda, 14 deles não apresentavam conhecimentos prévios quanto ao uso de 
softwares para análise qualitativa. Os demais afirmaram conhecer de forma elementar: Qualiquantsoft ${ }^{2}$, Nvivo ${ }^{3}$, Evoc ${ }^{4}$ e ATLAS.ti.

\subsection{Instrumentos}

Os instrumentos para coleta de dados foram : questionários (inicial e final), relato de experiência prévia com pesquisa, entrevista estruturada, grupo focal e diário de aprendizagem. Cada um desses instrumentos foi descrito detalhadamente, considerando não apenas que sua variedade permite a produção de dados de forma mais fidedigna ao fenômeno estudado, mas também lembrando que a variedade de instrumentos não pode tornar-se uma carga para os participantes (Lankshear \&Knobel, 2008).

Para a elaboração do questionário inicial, estabelecemos como objetivo uma avaliação diagnóstica acerca da experiência prévia dos estudantes. A organização desse instrumento compreendeu a formatação de um total de oito perguntas abertas e fechadas, abordando as experiências prévias dos estudantes quanto à pesquisa em geral, à pesquisa qualitativa e ao uso de softwares para análise de dados quantitativos ou qualitativos. Sua aplicação ocorreu no primeiro dia de aula, por meio do Qualtrics Survey Software ${ }^{5}$.

Ainda como avaliação diagnóstica para continuidade da pesquisa, escolhemos o relato de experiência, instrumento que consistiu em uma redação individual de cada um dos 25 estudantes participantes sobre sua trajetória e experiência com a pesquisa qualitativa, realizada no primeiro dia de aula, e compartilhada eletronicamente por $e$-mail. Assim, todos os estudantes participantes foram estimulados a conhecer o grupo que compunha o seminário. A esse respeito, Lankshear e Knobel (2008) dissertam que os registros que coletam dados escritos do ponto de vista do participante podem oferecer esclarecimentos úteis sobre seus pensamentos, bem como permitem reflexões posteriores.

A entrevista estruturada foi realizada em sala de aula, usando dispositivo móvel, de modo que os estudantes entrevistaram um ao outro. $\mathrm{O}$ entrevistado respondeu de forma sucinta à seguinte questão: de que forma sua formação acadêmica limitou ou contribuiu em sua formação como pesquisador?

Para complementar a produção dos dados, foi realizado um grupo focal em única sessão, com duas horas de duração. Essa técnica de entrevista em grupo permite a interação mais próxima da vida cotidiana (Flick, 2009), cuja principal vantagem reside no fato de os participantes estimularem um aos outros, a lembrarem de acontecimentos, ampliando

${ }^{2}$ Software de apoio a pesquisas qualiquantitativas, com base na teoria do discurso do sujeito coletivo. Disponível em: <http://www.spi-net.com.br/html/software.html>. Acesso em: 1 out.2016.

${ }^{3}$ Permite análise qualitativa com métodos mistos de pesquisa. Disponível em: <http://www. qsrinternational.com/what-is-nvivo>. Acesso em: 1 out.2016.

${ }_{4}^{4}$ Software de apoio à pesquisa em representações sociais. Disponível em:<http://www. academia.edu/2226246/openEvoc>. Acesso em: 1 out. 2016.

${ }^{5} \mathrm{O}$ Qualtrics Survey Software é um sistema que permite a elaboração e aplicação de questionários em diferentes modalidades e formatos, possibilitando o acesso à análise de dados quantitativos dos dados produzidos pelos respondentes. Disponível em: <https://www.qualtrics.com/login/>.Acesso em: 30 set.2016.Licença institucional da Pontifícia Universidade Católica do Paraná (2015). 
assim, a resposta de um único entrevistado (Flick, 2009). O objetivo da utilização do grupo focal centrou-se em elencar expectativas em relação à pesquisa qualitativa a ser desenvolvida individualmente tanto no mestrado como no doutorado. A gravação em vídeo por meio de dispositivo móvel como técnica de registro para esta etapa mostrou-se mais adequada.

O diário de aprendizagem consistiu em relatos semanais sobre o processo de aprendizagem individual, expressando expectativas e frustrações referentes aos temas abordados. Dessa forma, o diário foi construído, no decorrer de cada encontro, totalizando 12 relatos de aprendizagem em cada diário.

Por fim, o questionário final, no modelo survey, foi elaborado com cinco perguntas relacionadas ao processo individual de aprendizagem de cada participante do seminário, sendo quatro perguntas abertas e descritivas e uma fechada. Esse instrumento foi respondido por 12 estudantes.

\subsection{Exemplificação da aplicação da associação das técnicas de codificação}

Bardin (2010) propõe iniciar a análise dos dados pela leitura flutuante, que compõe a etapa de pré-análise. Contudo, conforme comentado, a análise qualitativa apresenta etapas e fases que não precisam necessariamente ser respeitadas sequencialmente. Percebemos, na fase exploratória, que antes de elencar códigos (Saldaña, 2013) e/ou indicadores (Bardin, 2010), foi necessário conhecer nosso corpus, para além da leitura flutuante, por meio da seleção de extratos ${ }^{6}$ livres, denominados por Saldaña (2013) de códigos in vivo, que serviram de base para a descrição e elaboração das memos analíticas.

Esse processo foi realizado com questionário do tipo survey, com base na perguntaproblema que nos norteava. Selecionamos, então, todos os extratos que detinham relação com o que buscávamos como resposta ou explicação para nosso questionamento. A Figura 2 exemplifica como destacamos os extratos das respostas no ATLAS.ti .

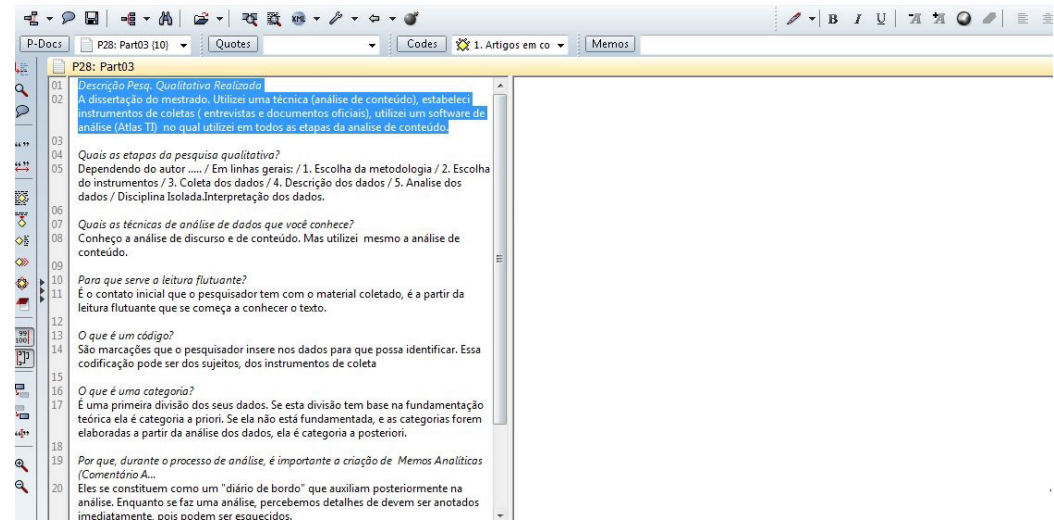

Figura 2 - Destaque de extratos no ATLAS.ti

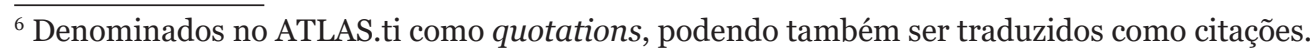


Com a finalização da seleção, foi possível visualizar todos os extratos e realizar uma leitura específica e detalhada apenas do material selecionado. A visualização da execução desse procedimento é possível por meio do gerenciador de quotations no ATLAS.ti, conforme mostra a Figura 3.

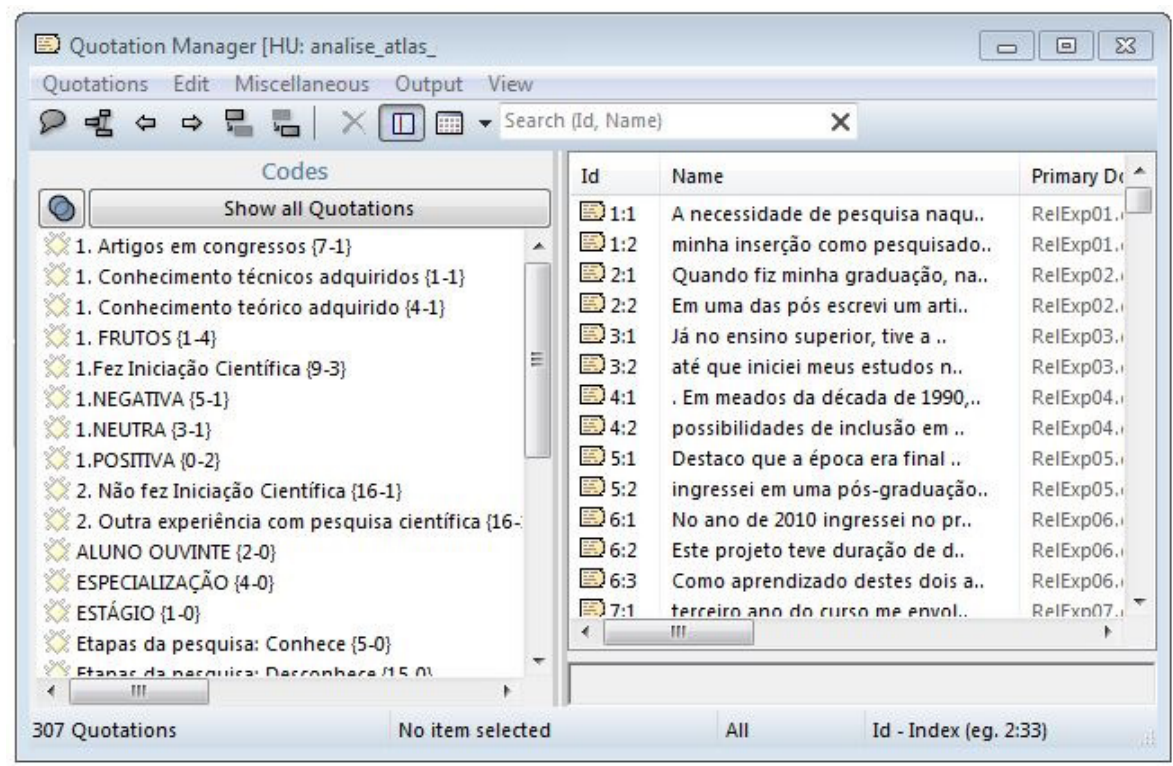

Figura 3 - Exemplo de quotations selecionadas no ATLAS.ti

Finalizadas essas etapas, passamos para as memos analíticas. É importante observar que a memo analítica evidencia a interpretação dos pesquisadores acerca de um trecho completo ou de parte do texto, utilizando os relatos de experiência e diários de aprendizagem. As memos ainda podem ser baseadas em citações teóricas ou mesmo citações de autores que embasam ou podem embasar a análise. Essa etapa é fundamental para que pesquisadores possam se familiarizar com o corpus de dados, bem como tecer breves análises, conforme apresenta o exemplo da Figura 4, elaborada por meio da utilização do ATLAS.ti.

A partir da memo analítica, identificamos que a estratégia de análise de dados deveria ser de acordo com a natureza de cada um dos instrumentos (questionários, relato de experiência, entrevista gravada, grupo focal e diários de aprendizagem). Dessa forma, sua produção foi de extrema relevância, permitindo detectar elementos que pudessem indicar a escolha de estratégias e/ou recursos diferenciados do software. Com isso, não houve perda de tempo com tentativas frustradas de análise, tempo precioso quando se trata de pesquisa qualitativa.

Só então iniciamos a fase de codificação, com base em Saldaña (2013), escolhendo a codificação descritiva para nosso primeiro ciclo de codificação. Para o autor, a codificação descritiva corresponde ao resumo em uma palavra, uma frase curta ou um substantivo do conteúdo referente a uma passagem dos dados qualitativos (Saldaña, 2013). É possível 


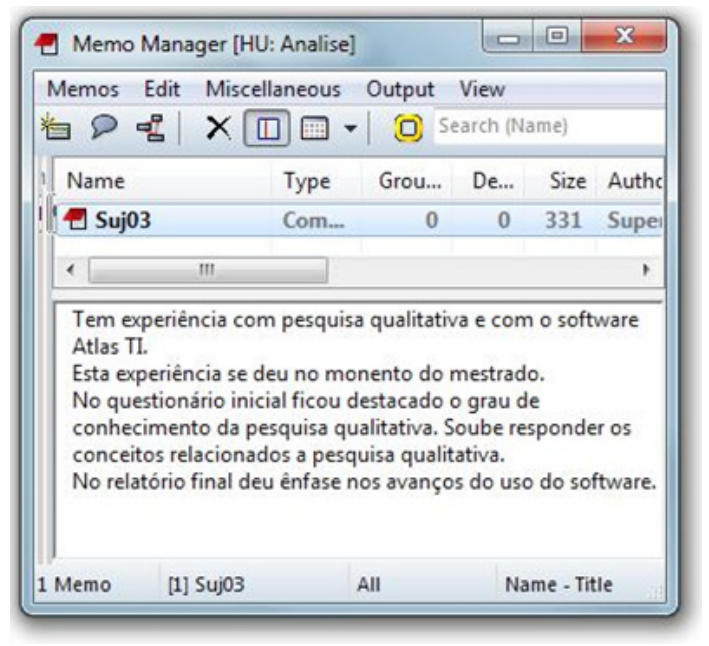

Figura 4-Memo analítica

verificar a quantificação das reincidências de nossa codificação tanto de forma descritiva quanto quantitativa na Figura 5.

\begin{tabular}{|c|c|c|c|c|c|c|}
\hline \multicolumn{4}{|l|}{ * Code Manager [HU: analise_atlas } & \multicolumn{3}{|c|}{ 回 83} \\
\hline \multicolumn{7}{|l|}{ Codes Edit Miscellaneous Output View } \\
\hline \multicolumn{7}{|c|}{ 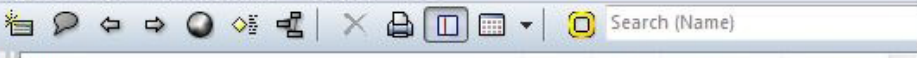 } \\
\hline Name & Gro... & De... & Author & \multicolumn{3}{|c|}{ Creater - } \\
\hline ( $\$ 2$ : 1, Artigos em congressos & 7 & 1 & Super & \multicolumn{3}{|c|}{$07 / 01 / 20 .}$. \\
\hline \% 1 . Conhecimento técnicos adquiridos & i & 1 & Super & \multicolumn{3}{|c|}{$07 / 01 / 20$} \\
\hline \% 1 Conhecimento teobrico adquirido & i & 1 & Super & \multicolumn{3}{|c|}{$07 / 01 / 20}$. \\
\hline 政1. FRUTOS & i & 4 & Super & \multicolumn{3}{|c|}{$07 / 01 / 20 .}$. \\
\hline 1.Fez Iniciação Científica & i & 3 & Super & \multicolumn{3}{|c|}{$07 / 01 / 20}$. \\
\hline 1.NEGATIVA & i & 11 & Super & \multicolumn{3}{|c|}{$07 / 01 / 20$} \\
\hline \% 1.NEUTRA & 1 & 1 & Super & \multicolumn{3}{|c|}{$07 / 01 / 20 . . \equiv$} \\
\hline 1.POSITVA & 0 & ii & Super & \multicolumn{3}{|c|}{$07 / 01 / 20}$. \\
\hline 2. 2. Não fez Iniciação Científica & 16 & 1 & Super & \multicolumn{3}{|c|}{$07 / 01 / 20 .}$. \\
\hline 2. Outra experiência com pesquisa científica & 16 & 1 & Super & \multicolumn{3}{|c|}{$07 / 01 / 20 .}$. \\
\hline 2 aluno ouvinte & 12 & 0 & Super & \multicolumn{3}{|c|}{$07 / 01 / 20}$. \\
\hline Zू: ESPECTALIZACCÃO & i & 0 & Super & \multicolumn{3}{|c|}{$07 / 01 / 20}$. \\
\hline \% ESTAGIO & i & 0 & Super & \multicolumn{3}{|c|}{$07 / 01 / 20 .}$. \\
\hline Z Etapas da pesquisa: Conhece & $\gamma$ & 0 & Super & \multicolumn{3}{|c|}{$08 / 01 / 20$.. } \\
\hline 2. Etapas da pesquisa: Desconhece & 15 & 0 & Super & \multicolumn{3}{|c|}{$08 / 01 / 20}$. \\
\hline Etapas da pesquisa: Superficial & 5 & 0 & Super & \multicolumn{3}{|c|}{$08 / 01 / 20 .}$. \\
\hline Fomestrado & 7 & 0 & Super & \multicolumn{3}{|c|}{$07 / 01 / 20}$. \\
\hline \% PROJETOS FORA DA ACADEMIA & 12 & 0 & Super & \multicolumn{3}{|c|}{$07 / 01 / 20$. } \\
\hline z.Descrição Pesq. Qualitativa Realizada & 21 & 0 & Super & \multicolumn{3}{|c|}{$08 / 01 / 20}$. \\
\hline \multirow{2}{*}{\multicolumn{2}{|c|}{ 2\%: z.O aue é coocorrência entre códiaos? }} & 0 & Super & & \\
\hline & & & & \multicolumn{3}{|c|}{ 08/01/20.. } \\
\hline No item selected & & & vatic - & & & \\
\hline
\end{tabular}

Figura 5 - Codificação descritiva 
Finalizada essa etapa, geramos as redes semânticas que permitem descrever os resultados prévios da codificação empregada. Destacamos que, ainda nessa etapa, é possível iniciar uma nova etapa de codificação, ainda do primeiro ciclo de codificação, por meio dos métodos gramatical, elementar, afetivo, literário, exploratório ou de procedimento (Saldaña, 2013). A escolha da tipologia de codificação depende da pergunta de pesquisa a ser respondida.

É importante observar, na Figura 6, que o método de análise empregado, bem como a codificação descritiva, permite a seleção de todos os trechos de texto com relação às questões e aos demais dados. Nesse caso, selecionamos a questão: "quais são as etapas da pesquisa qualitativa?”, referente ao questionário inicial.

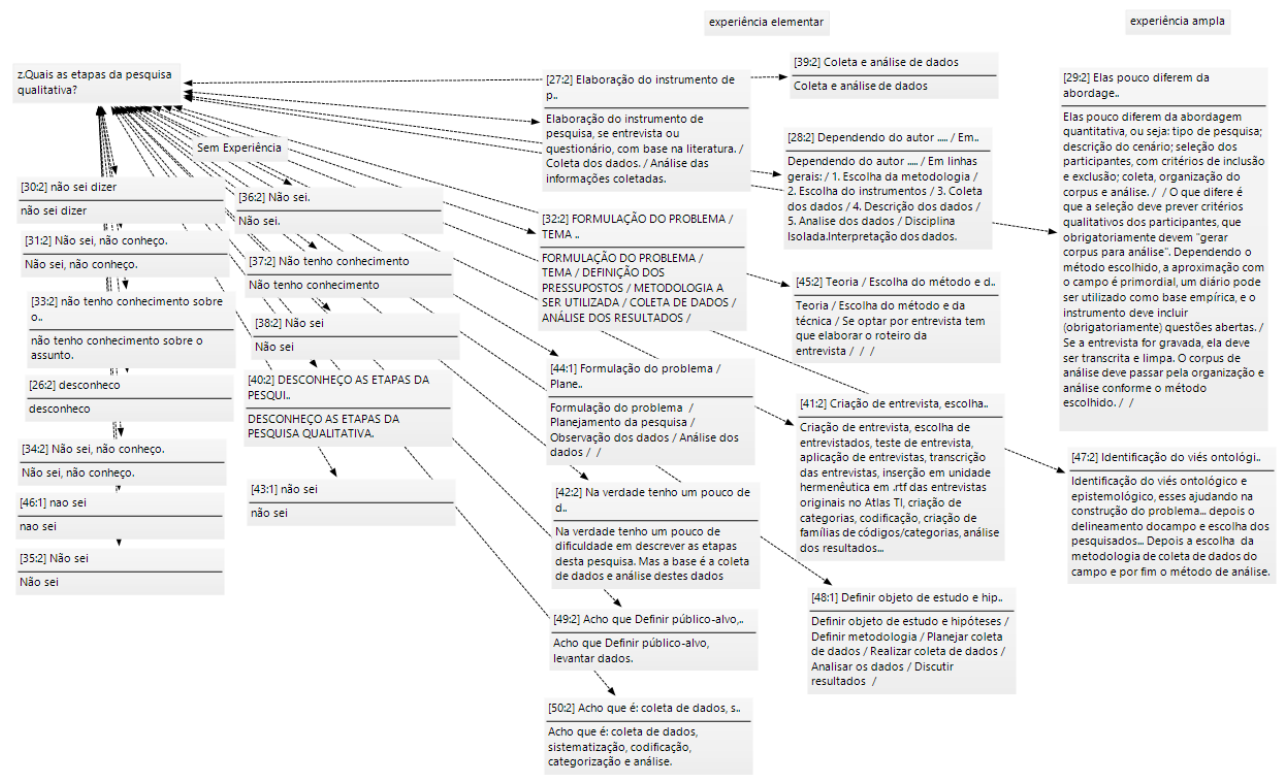

Figura 6 - Rede semântica a partir de uma questão do questionário

Após todo o processo de codificação descritiva, mostrou-se mais adequada a hierarquização dos extratos de cada código descritivo, ou seja, a aplicação de uma codificação por magnitude, Esse procedimento só pôde ser executado empregando-se um novo ciclo de análise, ainda dentro do primeiro descrito, o que permite exemplificar a codificação por magnitude a partir de Saldaña (2013), em complementação à técnica de codificação de Bardin (2010).

Para Saldaña (2013), a codificação por magnitude consiste em atribuir intensidade à avaliação do conteúdo ou uma sequência progressiva alfanumérica. Além disso, a magnitude pode ser expressa de forma quantitativa ou qualitativa. Em síntese, o exemplo evidenciado na Figura 6 mostra que a codificação por magnitude expressa-se de forma qualitativa, usando a avaliação do conteúdo extraído da codificação descritiva inicial. Nesse caso, os códigos podem ser descritos como: sem experiência (primeira coluna da 
rede semântica), experiência elementar (segunda coluna) e experiência ampla (última coluna). Esse exemplo revela a contribuição da complementação das técnicas de Bardin (2010) e Saldaña (2013), principalmente, quando os pesquisadores desejam trabalhar rigorosa e exaustivamente sobre o corpus de dados, mesmo que tenham recebido codificação e análise anteriores.

\section{Conclusões}

Iniciamos nossa pesquisa com várias questões compondo nossolabirinto de possibilidades de análise de dados em pesquisa qualitativa, que levaram a elaborar a pergunta-base pela qual guiamos nosso estudo: de que forma as etapas da análise de conteúdo de Bardin (2010) podem ser complementadas pelos ciclos de codificação de Saldaña (2013) e implementadas com o auxílio do software de análise de dados qualitativos ATLAS.ti?

Consideramos que nossa trajetória de construção dos instrumentos e a respectiva análise conduziu-nos a um caminho composto por quatro passos oriundos das bases teóricas referenciais, quais sejam: leitura flutuante; seleção das citações mais relevantes e congruentes com a pergunta norteadora; escrita de memórias analíticas relacionando as interpretações dos pesquisadores e sua relação com o referencial teórico; e, por fim, codificação em ciclos.

Este estudo mostrou a utilização de um ciclo de codificação por meio do método gramatical, com codificação descritiva e por magnitude. Dessa forma, pretendemos auxiliar outros colegas pesquisadores a saírem do labirinto de possibilidades, propondo mais um trajeto possível.

\section{Referências}

Alarcão, I. (2014). "Dilemas" do jovem investigador: dos "dilemas" aos problemas. In A. P. Costa, F. N. Souza, \& D. N. Souza (Ed.).Investigação qualitativa: inovação, dilemas e desafios (p. 103-123). Lisboa: Ludomedia.

Bardin, L. (2010). Análise de conteúdo. (L. A. Reto \& A. Pinheiro, Trad.). Lisboa: Edições 70,

Flick, U. (2009). Introdução à pesquisa qualitativa (3a. ed.). Porto Alegre: Artmed.

Lage, M. C. \& Godoy, A. S. (2008). O uso do computador na análise de dados qualitativos: questões emergentes. Revista deAdministração Mackenzie, 9(4), 75-98.

Lankshear, C. \& Knobel, M. (2008). Pesquisa pedagógica: do projeto à implementação. Porto Alegre: Artmed.

Leite, P., Gonçalves, J., Teixeira, P., \& Rocha, Á. (2014). Towards a model for the measurement of data quality in websites. New Review of Hypermedia and Multimedia, 20(4), 301-316.

Richardson, R. (1999). Pesquisa social: métodos e técnicas. São Paulo: Atlas.

Saldaña, J. (2013). The coding manual for qualitative researchers. London: Sage. 
Souza, N. S., Souza, F. N., Alarcão, I.,\& Moreira, A. (2015). Visão de orientadores e orientandos sobre o Software Online de Supervisão da Investigação - IARS®. RISTI - Revista Ibérica de Sistemas e Tecnologias de Informação, (E4), 66-78. http://dx.doi.org/10.17013/risti.e4.66-78

Teixeira, R. T., Mayr, L.R., Paisana, A. V. \& Vieira, D. (2014). Escolhas metodológicas em investigação científica: aplicação da abordagem de Saunders no estudo da influência da cultura na competitividade de clusters. RISTI - Revista Ibérica de Sistemas e Tecnologias de Informação, (E2), 85-98.

Varguillas, C. (2006). Usando Atlas.ti e criatividade do investigador na análise de conteúdo qualitativa. Journal of Education, 12, 73-87. Disponível em: <https:// www.uam.es/personal_pdi/stmaria/jmurillo/Met_Inves_Avan/Materiales/ Varguillas.pdf $>$. Acesso em: 1 maio 2016.

Vosgerau, D. S. R., Meyer, P. \& Contreras, R. (2016). Análise de dados qualitativos nas pesquisas sobre formação de professores. Revista Diálogo Educacional (no prelo).

Vosgerau, D. S. R., Pocrifka, D. H. \& Simonian, M. (2016). Etapas da análise de conteúdo complementadas por ciclos de codificação: possibilidades a partir do uso de software de análise qualitativa de dados. In Anais do CIAIQ2O16 (789-798). Porto: ULP. Disponível em:<http://proceedings.ciaiq.org/index.php/ciaiq2016/ article/view/671>. Acesso em: 1 set. 2016. 